\title{
Educación intercultural bilingüe. Un acercamiento a través de la práctica docente de las escuelas de la región Wixarika en el estado de Jalisco-México
}

\author{
Martha Vergara Fregoso*
}

Recibido: 19 de marzo de 2010 Revisado: 26 de marzo de 2010 Aprobado: 15 de junio de 2010

\section{Resumen}

El propósito de este artículo es compartir la experiencia de investigación y algunos hallazgos en cuanto al cómo se lleva a cabo la educación intercultural bilingüe en las escuelas ubicadas en la zona Wixarika-Huichola; comunidad indígena que habita en la zona norte del estado de Jalisco-México. Dicha investigación, titulada "Condiciones y mejoramiento de la educación intercultural de los estudiantes indígenas, nahuas y huicholes de educación primaria en Jalisco", fue financiada por los fondos SEP-SEByNCONACYT 2003-C01-451. La realización de la investigación se encaminó a la comprensión de los procesos y factores que entran en juego para determinar la calidad de la educación intercultural en la comunidad wixarika en el estado de Jalisco-México. Los indicadores de estas comunidades apuntan a índices altos de fracaso, de deserción y de reprobación escolar, así como la baja eficiencia terminal en las escuelas ubicadas en las dos zonas indígenas de referencia.

\section{Palabras clave}

Escuelas indígenas, educación intercultural, educación bilingüe, comunidad wixarika, indígenas mexicanos.

* Doctora en Educación de la Universidad La Salle. Miembro del SNI, del COMIE y de la REDMIIE. Actualmente se desempeña como profesora-investigadora en el Departamento de Estudios Internacionales de la Universidad de Guadalajara. Correo electrónico: mavederu@ yahoo.com.mx

1 El equipo de investigación estuvo integrado por: Martha Vergara Fregoso, como coordinadora de la investigación, y Gerardo Bernache Pérez, Ilda Esparza Martínez, María Guadalupe Cortés Godínez y Manuel Alcázar Cruz, como investigadores. 


\title{
Bilingual intercultural education. An approach through teaching practice in schools in the region Wixarika in the state of J alisco, Mexico
}

\author{
Martha Vergara Fregoso
}

\begin{abstract}
The purpose of this article is to share the experience of research and some findings as to how intercultural bilingual education is conducted in schools located in the Wixarika-Huichola; area an Indian community living in the northern state of Jalisco Mexico. This investigation, in titled "Conditions and improvement of the intercultural education for indigenous students, Nahua and Huichola Indians of Jalisco primary education", was financed by SEP-SEByN- CONACYT 2003-C01-45 funds. The conduct of the investigation was aimed at understanding the processes and factors that play a role to determine the quality of intercultural education in the community wixarika in the state of Jalisco, Mexico. The indicators of these communities shau high rates of school failure, dropout and low completion rates in schools located in both indigenous areas of reference.
\end{abstract}

\section{Key words}

Native schools, intercultural education, bilingual education, community wixarika, Mexican natives. 


\section{INTRODUCCIÓN}

Este trabajo forma parte de una investigación de mayor envergadura que surge a partir de la revisión de las estadísticas de la Dirección de Educación Indígena en Jalisco, cuyos resultados develan un alto índice de reprobación y deserción así como un bajo nivel de eficiencia terminal en los niños de educación primaria de las escuelas de los nahuas y huicholes.

Dadas las condiciones de desigualdad en el acceso a la educación básica, prevalecientes en algunas regiones del Estado de Jalisco, sobre todo en las rurales e indígenas, existe el riesgo de que se profundice la brecha entre los que reciben y los que aún carecen de servicios educativos de calidad.

Al respecto, Chavoya (1998) menciona que los niños del medio rural, y sobre todo los indígenas, llegan a la escuela con grandes desventajas: tienen una mala nutrición que puede afectar su capacidad de dedicar tiempo para aprender, no hablan el idioma de enseñanza, no entienden el código lingüístico usado en la transmisión de la educación, no disponen de materiales interesantes ni adecuados a sus necesidades, son atendidos por profesores sin la formación pedagógica mínima necesaria para ofrecerles buenas experiencias de aprendizaje...

En este mismo sentido, Muñoz (1999) afirma que

la educación de las poblaciones indígenas todavía no tiene el status de cuestión principal en la sociedad mexicana actual, a pesar de que constituye una demanda permanente de las organizaciones comunitarias e indígenas. Las discusiones sobre la educación bilin- güe han resultado menos influyentes que la doctrina de la modernización de la educación básica y también menos interesantes que la pugna por la hegemonía de ciertos paradigmas pedagógicos (didáctica crítica, constructivismo cognitivo, etnografía educativa, por ejemplo). Es entonces válido y necesario preguntarnos qué consecuencias tiene esta disparidad de criterios para el futuro de la educación en las escuelas indígenas mexicanas (p. 27).

Por lo anterior, los objetivos de la macroinvestigación fueron los siguientes:

- conocer las condiciones sociales e institucionales de las escuelas que atienden a la población indígena en el estado, así como entender las prácticas docentes y las acciones de los agentes educativos que participan en la educación primaria en comunidades indígenas de Jalisco;

- comprender los factores y las condiciones que determinan el rezago escolar de un sector importante de la población de alumnos de primaria en esta zona;

- identificar los factores que inciden sobre la baja calidad y los resultados pobres de la educación indígena, y

- contribuir a desarrollar estrategias para mejorar la educación en las comunidades huicholes de la zona norte.

En el presente documento se muestra parte del trabajo realizado para entender las prácticas y los significados que tienen los profesores que se desempeñan como tal en las comunidades primarias huicholes en Jalisco.

\section{Antecedentes}

El trabajo directo con profesores permitió recuperar información a través de varios acercamientos: un cuestionario, una entre- 
vista individual a profundidad (la cual fue audiograbada) y una entrevista aplicada inmediatamente al finalizar la clase. El interés inicial de la investigación fue evaluar las repercusiones de la práctica y los significados de los educadores que atienden estudiantes indígenas en el éxito y fracaso escolar, así como las condiciones límites y posibilidades de la educación intercultural bilingüe.

A partir de lo anterior, se formularon las siguientes preguntas:

- ¿Cómo son las prácticas de los profesores que laboran dentro de las escuelas primarias de educación intercultural bilingüe en la zona indígena huichol del estado de Jalisco?

- ¿Cuáles son los significados que tienen los profesores respecto a la práctica docente?

- ¿Cuáles son las condiciones, los límites y las posibilidades que prevalecen en las escuelas de educación intercultural bilingüe?

- ¿Cuáles son las estrategias que utilizan los profesores para el desarrollo de su práctica docente?

En el presente trabajo, se pretende la exposición, de manera muy general, de los significados que tienen acerca de la práctica docente, así como la descripción de las estrategias pedagógicas que utilizan para el logro de la educación intercultural, los profesores en estudio de las escuelas de la zona huichol del estado de Jalisco-México, y finalmente se vislumbran algunas reflexiones y recomendaciones para la mejora de la práctica docente.

\section{Perspectiva teórica $Y$ METODOLÓGICA DEL OBJETO DE ESTUDIO}

Dado que el medio indígena tiene su propia cultura, la educación que se imparte deberá ser vista como un fenómeno cultural; en este sentido, la cultura consiste en una red compleja de símbolos que provee de significados compartidos a los grupos y a las comunidades. Esa red de significados expresados en su red simbólica es conservada, elaborada y transmitida a las generaciones sucesivas, lo cual contribuye a un marco común de interpretación. Los sistemas simbólicos que se utilizan para construir el significado son sistemas preexistentes arraigados a la cultura y al lenguaje (Geertz, 2000). Constituyen herramientas comunales, que al usarse hacen del usuario un reflejo de la comunidad. Esos "sistemas simbólicos compartidos, de formas tradicionales de vivir y trabajar juntos" constituyen la cultura humana (Bruner, 1990, p. 28). En concreto, para Pérez (1998), la cultura consiste en un
conjunto de significados, expectativas y comportamientos compartidos por un determinado grupo social, que fa- cilitan y ordenan, limitan y potencian, los intercambios sociales, las produc- ciones simbólicas y materiales y las realizaciones individuales y colectivas dentro de un marco espacial y tempo- ral determinado (p. 16).

Al proceso de apropiación de la cultura (símbolos con significados) se le ha denominado socialización, consistente en el proceso de aprendizaje de los significados culturales que ayudan a los agentes a establecer y reproducir redes de relaciones sociales: familia, amigos, trabajo, etcétera. Así, un individuo socializado, además de ser capaz de interpretar adecuadamente los símbolos 
culturales propios del colectivo al que pertenece (reglas, creencias o valores), es capaz de integrarse a diversos grupos y vivir en sociedad (Essomba, 1999; Sacristán, 2001), y no sólo eso, sino que, de acuerdo con Pérez (1998), “vivir una cultura supone reinterpretarla, reproducirla tanto como transformarla" (p. 17).

De lo anterior se desprende que cada uno de los grupos culturales posee su propio sistema simbólico y de significados, es decir, sus propias reglas, motivaciones, creencias y actitudes que les hace pensar, sentir y actuar de un modo parecido y distinto a los otros. Esos contenidos constituyen la identidad de cada grupo, etnia, raza, región o nación; lo diferencian de los otros (Sacristán, 2001).

Dado el complejo objeto de investigación y los supuestos de los que se parte, el proceso de indagación está situado en la fenomenología, que como postura epistemológica se ocupa de describir cómo se constituye el mundo y cómo se experimenta a través de actos conscientes, lo que es dado en la experiencia inmediata sin estar obstruido por preconcepciones y nociones teóricas; se pregunta sobre la verdadera esencia de algo tal como se vive (Van Manem, 2003, p. 195).

Es una metodología descriptiva o fenomenológica, ya que

presta atención al modo en que las cosas aparecen, las cosas hablan por sí mismas, y es hermenéutica interpretativa- que afirma que todo fenómeno es interpretado. Los hechos de la experiencia vivida son experimentados de forma significativa y expresados e interpretados a través del lenguaje (Van Manem, 2003, p. 196).
Entiendo que no se trata de realizar descripciones directas de las manifestaciones del fenómenos, sino que "la reflexión fenomenológica radica en intentar aprehender el significado esencial de algo [...] es efectuar un contacto más directo con la experiencia tal y como se ha vivido" (Van Manem, 2003, p. 95).

La metodología seleccionada fue, en un primer momento, cualitativa y descriptiva, y posteriormente, interpretativa. Es cualitativa en virtud de que el objeto de indagación es la inferencia de los significados y la interpretación de las acciones y de la cultura enraizada en ellas por los actores educativos: docentes, autoridades escolares, padres de familia y niños indígenas.

El diseño se realizó en cinco etapas, las cuales se desarrollaron durante tres años comprendidos de enero de 2004 a diciembre de 2006. En la primera etapa, se llevó a cabo la selección de la muestra, conforme a los criterios del desempeño escolar de los alumnos (reprobación, deserción y eficiencia terminal). Se identificaron tres escuelas con mejor desempeño y once escuelas con desempeño bajo, las cuales se distribuyeron de manera proporcional, quedando cuatro escuelas de la zona sur (comunidades nahuas) y diez escuelas en la zona norte (comunidades huicholes). Además, se realizó una exploración del contexto y el primer acercamiento a los sujetos de estudio, mediante la aplicación de un cuestionario a 82 de 180 docentes, en la zona huichol, y 77 de 77 docentes, en la zona nahua.

En la segunda etapa, se realizaron entrevistas individuales a distintos actores y agentes de la educación indígena: directivos nacionales, equipo técnico estatal, dirigentes de la 
comunidad, padres de familia, profesores y alumnos profesores, padres de familia y algunos alumnos y alumnas; además, se levantaron registros de observación del contexto de la comunidad. Se utilizó la narración a través de entrevistas abiertas a los agentes de la población de la investigación como un medio de desplegar el significado, ya que "la narración trata del tejido de la acción y la intencionalidad humanas. Media entre el mundo canónico de la cultura y el mundo más idiosincrático de las creencias y deseos y las esperanzas" (Bruner, 2001, p. 63).

Durante la tercera, se llevaron a cabo observaciones y notas de campo sobre la práctica docente de los profesores. En ésta se recuperaron las acciones, especialmente del aula, con el propósito de lograr la saturación (Pérez, 1998). Se planteó levantar registros de aproximadamente tres horas cada uno en cada una de las aulas. Los registros son mediaciones pertinentes para describir las acciones y auxiliares en la interpretación de la cultura implícita en ellas. El análisis de los registros permitió conocer la práctica que realizan los profesores indígenas en las escuelas interculturales bilingües.

En la cuarta, se logró un acercamiento con algunos agentes, a quienes se les entrevistó: a la Dirección General de Educación Indígena de la SEP, a los integrantes del equipo técnico de la Dirección Indígena de la Secretaría de Educación Jalisco (SEJ), a un Supervisor y Jefe del sector de la zona norte de la Secretaría de Educación en Jalisco-México (SEJ).

Finalmente, en la quinta etapa, se llevó a cabo el análisis e interpretación de la información y presentación del informe de investigación. Cabe mencionar que, con el propósito de interpretar el corpus obtenido, se utilizó la hermenéutica, que es la teoría y la práctica de la interpretación. De acuerdo con Van Manem (2003, p. 44), "la interpretación es el acto de indicar algo (descubre aquello que el mismo objeto indica y lo que al mismo tiempo oculta) pero también es el acto de señalar el significado de algo (interpretamos una interpretación)". El conocimiento es una interpretación que parte de una situación social expresada en un texto (Van Manem, 2003, p. 197).

\section{LA PRÁCTICA DOCENTE COMO EJE DE LA INVESTIGACIÓN}

\section{El caso de los profesores huicholes-wixaritari}

Al hablar de la práctica docente se parte de reconocerla como una praxis social, objetiva e intencional en la que intervienen los significados, las percepciones y las acciones de los agentes implicados en el proceso (maestros, alumnos, padres de familia, autoridades educativas...; Fierro, 1999, p. 21); por ello, la práctica que realizan los profesores se caracteriza por ser dinámica (por sus constantes cambios), contextualizada (porque es in situ) y compleja (porque el entendimiento se da de acuerdo al tiempo y espacio); se considera además como una forma de la praxis, porque posee los rasgos de cualquier actividad: un agente ejerce su actividad sobre determinada realidad, con apoyo en determinados medios y recursos (Vergara, 2005).

La práctica docente se debe realizar desde el entramado que pudiera arrojar la observación y análisis cotidiano de ella, de los planes y programas que la enmarcan, de la 
visión que los diferentes sujetos involucrados tienen (maestro, alumno, directivos...), entre otras cosas. Para este documento, se hará desde la propia voz del docente y con relación sólo a algunos de los componentes de la práctica docente y situaciones problemáticas que se realizan.

Respecto a la práctica docente, ésta es definida por los profesores como todo lo que realizan en el salón de clases, lo cual incluye acciones realizadas antes, durante y después de la clase, como actividades dentro del salón y fuera de él. En este caso, está consciente de que la práctica no está determinada por los maestros, sino que ésta se va estructurando de acuerdo a cada situación y momento que se vive, la forma en que se debe tratar a los estudiantes, la metodología que se debe emplear y cómo el ser docente se va adquiriendo a través del tiempo.

De acuerdo a las entrevistas y observaciones de clase realizadas a los profesores en el aula, la práctica se refiere a lo que se realiza en el aula y el proceso enseñanza-aprendizaje. En este mismo sentido, a través del discurso de los profesores, encontramos otros significados relacionados con el profesor como persona, la figura del maestro, su función, finalidad, etc.

\section{Práctica docente $=$ el hacer en el aula}

En lo que respecta a la práctica docente investigada por Bernache, Esparza, Cortés y Alcázar (2005), los profesores refieren que ésta se relaciona con lo que realizan en el aula y en el proceso enseñanza-aprendizaje, tal como se muestra con los siguientes fragmentos: "Es todo lo que hace el docen- te en el proceso enseñanza-aprendizaje" $(\mathrm{C} 1 Z \mathrm{ZN} 23)^{1}$; “"es el quehacer docente en el aula y comunidad en donde está ubicada la escuela" (C1ZN25); "todo lo que hace el docente diariamente para su práctica cotidiana" (C1ZN27); "lo que hacen diariamente los docentes" (C1ZN53); "es el quehacer o el trabajo diario que realiza cada docente en el ámbito que le corresponde" (C1ZN61), y "son actividades rutinarias, lo que el maestro hace diariamente en el aula junto con sus alumnos" (C1ZN01).

Los significados que se pueden inferir a partir del discurso de los profesores es que la práctica docente la realiza un sujeto, que en este caso es el docente; en este sentido, coincide con Fierro, Fortul y Rosas (1999), quienes afirman que la práctica docente se realiza en una institución escolar (la escuela), la cual representa para el sujeto-docente el espacio de socialización profesional.

También se encuentran otros significados en la investigación de Bernache et al. (2005), que responden a las acciones que realizan los docentes, pero con una finalidad explícita: "la práctica docente consiste en desarrollar las actividades escolares mediante una planeación diaria, dentro del salón y fuera" (C1ZN39); "es la que realizo o llevo a cabo en mi trabajo cotidiano para el aprendizaje de mis alumnos" (C1ZN46); "es la que se lleva a cabo en las acciones de enseñanzaaprendizaje con los educandos mediante contenidos adecuados" (C1ZN24). En este mismo sentido, a través del discurso de los profesores, se encontraron otros significados relacionados con el profesor como persona.

1 El significado del código C1ZN23 es: C1 significa el cuestionario número uno, la $\mathbb{Z N}$ corresponde a la zona norte y el número al profesor que dio respuesta al instrumento. 


\section{El ideal del profesor}

De acuerdo con los entrevistados por Bernache et al. (2005), el profesor debe contar con algunas características personales, las cuales van desde el ejemplo que implica su persona y su función desde lo ideal: "es aquel que enseña con el ejemplo" (C1ZN20); "la paciencia es una de las virtudes que utiliza para la enseñanza”; "ayudar al que necesite de mis servicios" (C1ZN61); "ser sociable, tolerante" (C1ZN46); "tener una visión de lo que es la educación" (C1ZN27); "ser guía y transformador de pensamientos con valores éticos" (C1ZN51).

La vocación es un elemento recurrente en el discurso de los maestros, como un elemento constitutivo de todo(a) profesor(a), lo cual tiene mayor peso que la formación que puede llegar a tener; de acuerdo con lo indagado por Bernache et al. (2005): "una persona con vocación que desempeña innatamente su actividad, que orienta, instruye y educa" (C1ZN149); sin embargo, también aparece una contradicción con otros profesores, ya que está presente la importancia de la permanente búsqueda y una formación de calidad con el fin de lograr una mejor práctica docente; según un entrevistado por Bernache et al. (2005): “seguir preparándome profesionalmente y servir mejor en mi práctica docente, mostrar la suficiencia en la enseñanza, aumentar el nivel cultural, seguir descubriendo nuevos conocimientos" (C1ZN123).

A manera de definiciones, en cuanto se refiere al docente como tal, rescato las siguientes de Bernache et al. (2005): 1) “es un orientador, guiador del alumno, del padre de familia, en ocasiones de la comunidad y también formador del alumno para que enfrente su vida real" (C1ZN148) y 2) "en lo general me da la idea que maestro es una persona que desempeña un oficio funcional determinado, pero lo que respecta a maestro pedagogo, es con las cualidades y habilidades de enseñar al alumno moral e intelectualmente" (C1ZN124).

Alrededor de la figura educativa del maestro, se encontraron diferentes significados. Para algunos, el significado implica de por sí su responsabilidad y las actividades que desarrolla; por otro lado, implica las intenciones educativas que se plantean, los valores que promueve, hasta cuestiones más personales relacionadas con su conocimiento y sus actitudes.

Hablar del profesor es hacer referencia a la persona, ya que estos dos aspectos no se pueden separar; en este sentido, al docente se le atribuye una serie de responsabilidades que tienen que ver con su labor en la educación intercultural bilingüe, pero también algunas características que se relacionan con la figura del profesor.

\section{Entrevistador: ¿Cuál crees que es la responsabilidad de los profeso- res en la educación intercultural bilingüe? \\ Entrevistado: Cumplir con el hora- rio... cumplir con las fechas del ca- lendario, estar al servicio de la so- ciedad, apoyar a la sociedad para mejorar la educación como debe ser (ZNGUCT03) ${ }^{2}$.}

Mientras que en el discurso de otros profesores aparece la importancia que le dan a

2 Esta codificación (ZNGUCT03, por ejemplo) se utilizó para identificar la información proveniente de la aplicación de entrevistas individuales a los maestros participantes en la investigación. Las letras $\mathbb{Z N}$ representan la zona norte, las dos siguientes hacen alusión al nombre del lugar en el que se encuentra la escuela visitada, el siguiente par de letras contiene el nombre de escuela y, finalmente, el número es el asignado al profesor entrevistado. 
las reglas y normas del centro de trabajo, tal como se expone en el siguiente fragmento:

Entrevistador: ¿Cuáles son las responsabilidades del profesor, qué tiene que hacer, cómo lo tiene que hacer?

Entrevistado: Lo que tenemos que hacer como maestros, es ser responsables en nuestros centros de trabajo, invitar a que participen los jóvenes y todos los padres de familia.

Entrevistador: ¿A qué se refiere cuando dice que es ser responsable en el centro de trabajo?

Entrevistado: Se refiere a que la escuela tiene muchas reglas que debemos cumplir, pero que también debemos estar comprometidos con la demás gente (ZNLLCM05).

Sin embargo, un nuevo significado se puede inferir en el discurso de los profesores relacionado con un sentido de identidad, con el que se enfatiza que es necesario que el papel del profesor debe responder a lo que requiere la comunidad, ya que ellos se deben a los niños, pero también se justifican por no cumplir cabalmente con su función, debido a que requieren de ciertos apoyos.

Entrevistador: ¿Cuál crees que es el papel del profesor?

Entrevistado: Primero es que debemos ser responsables, uno debe uno sentirse como propio de la comunidad de los niños, ayudar, trabajar bien, a cumplir bien.

El problema siempre yo veo, que no aplicamos bien, o sea no sabemos estrategias de enseñanza, a ayudar a los niños. Sí tenemos material, bueno yo tengo muchos libros, los niños todos yo quiero como una capacitación, necesito la capacitación para saber cómo se usan los materiales, los libros, para así si me falta pues casi todo, así veo a mis compañeros, una capacitación de todo con lo que se mandan (ZNTBCU27).
Con todo lo anterior, se puede decir que, al hablar de la práctica docente, es necesario considerar que se trata de un concepto complejo que se constituye en torno al ejercicio de los profesores y que cada práctica es influida por los diferentes significados que estos han construido sobre ella, debido a que en la experiencia cotidiana del trabajo docente coexisten los elementos institucionales y personales del papel que desempeñan; así, cada maestro es diferente en su práctica profesional. Existe pues una gran diversidad de maneras de ejercer la docencia debido a los significados que construye cada sujeto sobre la práctica, los cuales se manifiestan en diferente grado o amplitud en las acciones que realizan (Vergara y Bernache, 2008).

Lo anterior resulta determinante para que cada profesor en las escuelas indígenas bilingües construya los significados de la práctica docente en torno a su formación y a su historia personal, ya que la manera como es vista la práctica docente se va construyendo a través del tiempo y de la interacción que el docente tiene con sus compañeros de la institución.

\section{Enseñar a los alumnos/alumnas}

En relación con los diferentes significados que tienen los profesores sobre enseñan$z a$, se puede ver que en todas las acepciones citadas existe una visión en la que se da a entender la presencia de dos sujetos, el que transmite un conocimiento o facilita el aprendizaje y el que lo adquiere o interactúa con el conocimiento hasta llegar al aprendizaje.

Entonces, el significado de aprendizaje se encuentra implícito en este concepto, pues 
se alcanza a visualizar como el fin de la enseñanza, aun sabiendo que hay diversas posiciones frente a éste (lo cual se verá más delante). Además de los actores involucrados, es palpable la presencia de elementos que resultan fundamentales: contenidos, actitudes, valores, habilidades, etc., que se pretende aprender o desarrollar, las cuales se vuelven el qué, el centro de ese encuentro entre sujetos en el proceso de aprendizaje.

\section{Interacción entre sujetos}

\section{involucrados en el proceso de enseñanza-aprendizaje}

De acuerdo con lo extraído por Bernache et al. (2005): "es la interacción educativa que comparten las personas que aprenden a modo de procesos o del contenido lo que se está desentrañando" (C1ZN30); "es una actividad en el maestro que facilita la construcción que realiza el alumno a través de su conocimiento" (C1ZN21); "es una actividad interactiva y de carácter procesal en la que el maestro facilita el conocimiento sobre el alumno" (C1ZN14).

Otro de los significados sobre la enseñanza que se puede inferir en el discurso de los profesores es el de transmisión de conocimientos. En este significado es común identificar que la transmisión se lleva a cabo a través de la figura del que más sabe, que en este caso es el profesor. Así, al preguntarles qué entienden por enseñanza, Bernache et al. (2005) lograron captar las siguientes respuestas: "es un proceso establecido que trata de transmitir un conocimiento de un maestro al alumno" (C1ZN04); "transmitir un conocimiento a los alumnos o a otras personas" (C1ZN); "es de transmitir a otros lo que uno sabe" (C1ZN11); "es un proce- so en donde se transmiten los conocimientos a las nuevas generaciones" (C1ZN61); "transmitir conocimientos a otras personas" (C1ZN04); "transmitir los conocimientos en forma verbal y escrita de lo que conoce uno como maestro" (C1ZN76); "transmitir un conocimiento con estrategias adecuadas" (C1ZN49); "transmitir conocimientos a los alumnos a través de actividades pedagógicas" (C1ZN22); "transmitir nuevos conocimientos a los niños" (C1ZN70).

De esta manera se puede inferir que tanto la educación como la enseñanza están vinculadas a la transmisión de conocimientos, en la que el papel del maestro consiste en ser transmisor y el del estudiante, en ser receptor de conocimientos. Aquí, el rol del docente consiste en dirigir las actividades que realizan los niños para lograr que ellos aprendan a través de la instrucción; el profesor es el centro de toda actividad educativa, por lo tanto, la enseñanza resulta un sinónimo de adquisición de conocimientos por parte del alumno.

Al respecto, Postic (2000) refiere (así como otros autores que coinciden con Cousiner) que: "Ser alumno en nuestro sistema actual es recibir informaciones, consejos, incitaciones que conciernen al aprendizaje que hay que realizar, pero también (y quizá sobre todo) es depender de un adulto que posee la autoridad, de un adulto que los juzga, que los estima y los persigue con sus amonestaciones o los ignora" (2000, p. 67).

Uno de los aspectos por los que se reconoce a un maestro o maestra es la responsabilidad que se le ha depositado históricamente de enseñar al(a la) alumno(a), en la que su posición es de superioridad, pues se le reconoce con un bagaje teórico, de forma- 
ción y experiencia que sobrepasa la de sus alumnos(as). En este sentido, es el poseedor del conocimiento que los otros y las otras tienen que conocer y aprender. Como se dice coloquialmente, es el director de la orquesta.

Aquí, la enseñanza se basa en el conocimiento del maestro, pero también en lo que los planes y programas señalan, ya que para ellos es importante cumplir con lo establecido en los planes y programas de estudio. Según se encuentra en Bernache et al. (2005): “enseñar, respetando los planes y programas, apoyar a la niñez en su aprendizaje, enriqueciendo sus conocimientos previos" (C1ZN76). De esta manera, la enseñanza de los alumnos también es entendida como una responsabilidad que tienen los docentes wixárikas. En ZNPNJS08 se aprecia: “Entrevistador: ¿Cuál es tu responsabilidad como docente? Entrevistado: Mi responsabilidad, es que primero hay que ser responsables en cuanto a la revisión de todos los contenidos que vas a abarcar durante todo el año".

Como se puede observar en el fragmento anterior, el significado de la responsabilidad se restringe a cumplir con las normas establecidas en cuanto a las fechas y al horario, entre otros aspectos, como los contenidos del programa.

Lo anterior contradice lo que afirma Segovia (1998), en cuanto al currículum educativo, lo cual se encuentra establecido en los planes y programas de educación primaria:

el currículum viene señalado por decisiones superiores que trascienden al propio profesor, y que éste debe seguir en su clase. Pero el profesor no puede seguir el currículum al pie de la letra como un guión que marca detallada e inexorablemente los pasos de la secuencia didáctica (p. 29).

El profesor de cualquier nivel educativo debe hacer uso del currículum como un recurso o amplificador de su propia competencia profesional. No se trata de que el profesor cuente con los conocimientos que va a impartir sino que lo más importante es la capacidad para abordar y lograr que los estudiantes se apropien de los contenidos. Otro de los significados inferidos es aquel en el que queda establecida cuál es la finalidad de la enseñanza, la cual, de acuerdo con el discurso de los profesores entrevistados, es lograr aprendizaje en los alumnos(as). En este sentido, el significado de la función del profesor cambia, pues el centro del proceso de enseñanza-aprendizaje no es él como sujeto poseedor del saber, sino el alumno, quien es el aprendiz; así, su responsabilidad es mediar el proceso de aprendizaje de sus alumnos(as). Se constata en lo que dicen los entrevistados por Bernache et al. (2005), por ejemplo, cuando dicen: "contribuir al desarrollo y mejora, en el aprendizaje de los alumnos" (C1ZN09) o "el maestro es el intermediario del plan y programa de estudio entre el niño que recibe el conocimiento" (C1ZN25).

De igual manera aparece entre otras finalidades de la enseñanza en el medio indígena, la cual se restringe a enseñar en la escuela para leer y escribir. Aquí el proceso de lectoescritura resulta uno de los aspectos más importantes por trabajar en términos de conocimientos $y$ habilidades para ser desarrollados por los niños y las niñas de la región, pues es uno de los elementos que precisan los(as) maestros(as) con mayor énfasis, seguramente por el valor de uso que representa y porque lo ven como un medio 
para llegar a aportar a su comunidad cuando sean adultos. Algunos entrevistados por Bernache et al. (2005) afirman: "enseñar a los alumnos a leer y escribir y sepan resolver sus propios problemas en el futuro" (C1ZN08) o "sacar los alumnos con nuevos conocimientos, leyendo y escribiendo, etcétera" (C1ZN40).

La lectoescritura es uno de los medios que permiten el diálogo y la relación con la cultura dominante, por eso se convierte en una de las herramientas formativas y de desarrollo prioritarias en la región. Para quienes llegan a tener cargos comunitarios, resulta indispensable dominar la escritura, principalmente en español, con el fin de poder comunicarse con las diferentes instancias, organizaciones, autoridades mestizas, etc. Así se ve en Bernache et al. (2005): “enseñar bien a los alumnos a que sepan leer, escribir, apoyar a la comunidad que se requiere y con el tiempo llegar a ser líder rural" (C1ZN15).

La lectoescritura también tiene un significado que se relaciona con los cimientos para una profesión, ya que si una persona llega a leer y escribir en castellano, entonces esto será la base para que pueda llegar a ser profesionista. "Formar alumnos con hábitos de leer, escribir y continúen sus estudios más adelante, hasta profesionistas" (En Bernache et al., 2005, C1ZN14).

\section{Respecto al aprendizaje, se tiene} que...

Los significados que tienen los profesores sobre el aprendizaje están estrechamente ligados a los inferidos en el concepto de enseñanza, ya que se ve como el resultado de dicho proceso y, concretamente, de la transmisión de conocimientos aprendidos en la escuela.

Los significados inferidos acerca del concepto de aprendizaje están relacionados con la adquisición de conocimientos, habilidades y hábitos que se pueden percibir en las acciones realizadas para acercarse a ese conocimiento; en la figura de la escuela como el lugar donde se lleva a cabo el aprendizaje, aunque en algunos profesores aparece el significado del aprendizaje significativo.

\section{Aprender para la adquisición de conocimientos}

En Bernache et al. (2005) se aprecian, por ejemplo, opiniones como: "adquirir algún conocimiento favorable para la vida" (C1ZN155); "adquirir un conocimiento de calidad para su futuro" (C1ZN54); "adquirir nuevos conocimientos para conocer otros nuevos modelos de aprendizaje" (C1ZN56); "es adquirir conocimiento nuevo" (C1ZN13); "es la adquisición de conocimientos, cualidades, actitudes y valores" (C1ZN21); "la adquisición de nuevos conocimientos y ampliar lo que se conoce, no olvidando la lectoescritura" (C1ZN08), entre otros. Por lo anterior se puede decir que el significado del aprendizaje está estrechamente relacionado con el resultado de la transmisión de conocimientos aprendidos en la escuela; por lo tanto, es a la vez el resultado de la enseñanza. En este sentido, se le atribuye a la escuela un papel importante en el aprendizaje, de tal manera que se coincide con Delval (2001) en que

la escuela lo que transmite más frecuentemente son los resultados del conocimiento científico, que sin em- 
bargo en esa forma de transmisión se convierten en algo fijo y definitivo. Los alumnos tienden a adquirirlo por medio de la repetición y la memorización de enunciados que frecuentemente no entienden (p. 37).

\section{Uso de materiales de apoyo a la enseñanza}

Entre las principales preocupaciones respecto a la práctica docente de los profesores en estudio está el cumplimiento de los planes y programas. En este sentido, para los profesores de la región Wixarika, es importante la adecuación de contenidos, estrategias, técnicas y actividades a las necesidades y al contexto de los alumnos(as), con el fin de construir significados en ellos y ellas para que el aprendizaje y desarrollo sea posible. Se puede constatar en las afirmaciones de estos dos entrevistados:

yo lo que hago es adaptar los contenidos y darlo en la lengua, lengua materna, porque en... primero y segundo, lo estoy dando en la lengua materna y en tercero ya se le intenta meter el español, pero en segundo se tiene que dar en lengua indígena y así nosotros lo estamos utilizando (ZNTXLC35).pues, sacar adelante los contenidos..., lo más importante, adaptarlos, pues, aquí en el medio. Yo digo, pues, que los niños hagan su narraciones, cuentos, o lo que hacen aquí los huicholes, pues, como por ejemplo, arcos, figuras de barro, así pues, para que rescaten su cultura (ZNTXLC37).

De igual manera, otra preocupación es el logro del bilingüismo, el uso de la lengua:

Encuestado. Bueno, en mi caso, en mi escuela, en cada ciclo escolar que se inicia, platicamos con los maestros de primero. Ellos anteriormente, comen- zaban con el huichol, así era: el huichol primero y ya de enero en adelante, comenzaban a dar lo que es el español; pero vimos pues, esa diferencia,... que venían los exámenes y caíamos en lo mismo. Entonces, ahora estamos al revés, eh..., ahora iniciamos con el español, ahora sí, utilizando los libros de texto y yo creo que en enero, febrero, más o menos, tendrían que enseñar lo que es el huichol, nada más el alfabeto y ya con eso, pero sí hay... y ya los demás grados, pues puro español. (ZNTXLC38).

Con todo lo anterior se puede afirmar que resulta importante para los profesores contar con los materiales de apoyo elaborados por la SEP, ya que no existe un plan y programa específico para la educación indígena, sino que ésta se opera de acuerdo al Plan y Programa de Educación Primaria de 1993. Entre los elementos que contiene este documento, están: el enfoque de cada una de las asignaturas y de las ocho asignaturas que se trabajan en este nivel educativo: matemáticas, español, ciencias naturales, historia, geografía, educación cívica, educación artística y educación física, incluidos en un sólo libro, el cual tiene la finalidad de que los profesores logren a través del análisis del documento, tener una visión de conjunto de los seis grados, motivo por el cual se pretende que los profesores conozcan "los propósitos y contenidos de todo el ciclo y no sólo de los que corresponden al grado en el cual enseñan" (Secretaría de Educación Pública [SEP], 1993, p. 8).

Con este antecedente, nos acercamos al aula de clases para conocer el uso que los profesores en estudio daban a los materiales educativos elaborados por la SEP y además para darnos cuenta de qué otros materiales echan mano los profesores. 
Los profesores reconocen que cuentan con algunos materiales de apoyo, elaborados por la SEP; sin embargo, en su discurso sólo aparece el conocimiento del Plan y Programa de Educación Primaria editado en 1993 y no hacen mención de otro tipo de documentos que apoyen a la educación intercultural bilingüe, como se constata a continuación:

Encuestador: ¿Con qué materiales de apoyo cuentas para el desarrollo de tu práctica?

Encuestado: Tengo el plan y programa de educación primaria... este libro sí se usa, sí lo conozco, sí entiendo allí, lo malo es que faltan cosas, hay muchos contenidos que no se adaptan, el programa casi no es realidad a las comunidades en que se vive, a las ciudades si se da, pero allá es muy diferente, como los niños no saben hablar castellano, no entienden lo que viene en los libros (ZNTBCU27).

En las entrevistas realizadas, los maestros de la zona huichol afirman que los programas de educación primaria refieren el uso de los libros de texto, sin embargo, estos no responden a su realidad porque muchos de los contenidos y/o temas son desconocidos por los niños ya que tienen dificultad para hablar el castellano. Por lo tanto, el uso que dan los profesores al Plan y Programas de Educación Primaria se refiere a continuación:

Encuestador: ¿En qué te apoyas y cómo haces uso del plan y programa de 1993? Encuestado: Siempre se nos ha exigido que debemos seleccionar los contenidos que vamos a trabajar de ese documento. Entonces me ha servido para seleccionar lo que vamos a trabajar. Lo único que hacemos nosotros es que los adaptamos de acuerdo a la región... aunque muchas veces eso no lo podemos hacer. Porque hay unos contenidos que vienen de acuerdo a como el niño se está desarrollando allá en la región mestiza y entonces es muy diferente en la región de acá, entonces nada más seccionamos contenidos sencillos y los adecuamos de acuerdo al contexto del niño (ZNPNJS07).

Con el fragmento anterior, se puede evidenciar que los profesores tienen claridad en cuanto a la necesidad de adaptar los contenidos establecidos en el Plan y Programas de la SEP de 1993 a las necesidades de sus alumnos, sin embargo, llama la atención la afirmación que realiza el docente en cuanto a "entonces nada más seccionamos contenidos sencillos y los adecuamos de acuerdo al contexto del niño". Ello muestra que la preocupación del profesor está en la selección de los contenidos por sencillos, más no por la utilidad que tengan estos en el desarrollo del estudiante.

Para llevar a cabo la planeación de cada una de las sesiones de clase, algunos de los profesores entrevistados dicen que toman en cuenta las actividades que van a realizar; para ello, dan mayor relevancia a las asignaturas de matemáticas y el español, porque consideran que éstas son las más importantes en el aprendizaje de los niños.

De igual manera, otro de los profesores en estudio que atiende los grados de primero y segundo en una de las escuelas afirma que los libros de texto son importantes porque le ayudan a llevar a cabo su práctica, ya que, a través de ellos, los niños pueden conocer algunas cosas que en la comunidad no tienen; sin embargo, es difícil explicarles a los niños la utilidad de esas cosas que vienen en los libros.

El uso que dan a los materiales bien puede ser de consulta o para hacer seguimiento 
a las actividades que deben realizar en el aula; tal es el caso de uno de los profesores entrevistados. Otros docentes reconocen la existencia de materiales diferentes al Plan y Programa, los cuales se dirigen a la atención de grupos específicos; tal es el caso de documentos para la atención a grupos multigrado.

Es común en varios de los profesores el uso de un libro de texto comercial, que de acuerdo con lo observado en las sesiones de trabajo y en la entrevista, utilizan para la realización de su trabajo docente. Es evidente que copian algunos ejemplos de ese libro, los cuales ponen luego a los alumnos para que den respuesta; sin embargo, lo más preocupante es que algunas veces estos textos son la base para el trabajo en el aula y dejan de lado los libros de texto editados por la SEP. El significado que tiene el libro comercial en algunos profesores es que les ayuda a elegir ejercicios más elaborados que los que podrían realizar ellos; tal es el caso de la profesora que dice: "el libro en el que me apoyo, me ayuda para tener más $\mathrm{y}$ diferentes ejercicios para que los alumnos no se aburran" (ZNTBCU40).

Para otros profesores, los libros comerciales tienen otro significado: "yo los utilizo porque me ayudan a hacer más simple la planeación que tengo que hacer, porque aquí ya viene todo en el orden que debe ser" (ZNTBCU43).

Esto refleja que el profesor sustituye el Plan y Programas Oficial, editado por la Secretaría de Educación en el país, por un libro editado por una casa comercial.

En el caso de una de las profesoras que laboran en la zona norte y que atiende el ter- cero y cuarto grados, los ejercicios que toma del libro comercial son para que los niños de ambos grados escolares den respuesta. Cabe hacer mención que este libro comercial sólo lo tienen los profesores y no los alumnos, por lo que los niños no conocen su contenido.

Otro de los profesores, el que es responsable de los grados primero, segundo y sexto, se apoya en otro libro comercial, recurriendo a ese material únicamente para el sexto grado.

\section{Estrategias pedagógicas para el logro de la educación intercultural}

Entre las estrategias que utilizan los profesores para trabajar la educación intercultural está la traducción que hacen algunas veces de los libros de texto, mientras que en otras se trabaja un tiempo con el Wixarika y luego con el español.

Encuestado: Estee [sic] entre las estrategias para trabajar ambas lenguas... pues es nada más traducir, de sacar algo de la región pero como le decía hace rato, es que pues, necesitamos... que tengamos ahora sí, registrado como asignatura pues, para que nosotros podamos dar bien las clases a los niños.

Entre las estrategias utilizadas por una de las docentes de segundo grado, se encuentra la de realización de preguntas a los estudiantes. Estas preguntas, de acuerdo a la entrevista realizada, propician la participación del alumno, ya que es una manera de atraer su atención y saber qué tanto conocimiento posee sobre el tema tratado. Sin embargo, en este caso, la estrategia no 
logró tomar en cuenta las aportaciones de los alumnos, lo que produjo que se perdiera el interés. De esta manera, por ejemplo, en una oportunidad, la maestra inicia la clase pidiendo a sus alumnos que le digan varios números, los niños contestan; luego escribe cuatro cantidades en el pizarrón que no corresponden a las que dijeron los alumnos; los chicos siguen diciendo cantidades y ella les dice que ya es suficiente y anota otras cantidades que no fueron expuestas por los estudiantes. El entusiasmo del alumno por colaborar y participar en clase se manifiesta al pasar al pizarrón y al responder acertadamente el cuestionamiento de la docente, situación que denota la existencia de un aprendizaje. Sin embargo, estas acciones no son consideradas de esa manera por la profesora. Al terminar la suma, la docente les pide que saquen su libro de matemáticas:

\section{Maestra: En la 101 de matemáticas... ya todos están en la 101, ahí vamos a con- tar, vienen unos dibujos ¿Cuántos son aquí? (señalando el libro de texto). Alumnos: Veinticuatro. \\ Maestra: En la que sigue (ZN2-230506).}

Como se observa en el párrafo anterior, los estudiantes responden las cuestiones que les solicita su maestra; ella escribe en el pizarrón el esquema que completarán los alumnos en el pizarrón y comienza a explicar cómo deben trabajar. Los niños comienzan a trabajar y la titular observa a cada uno para prestar su apoyo. Algunos se acercan a su escritorio para que les revise el trabajo realizado. Durante la actividad, dos niños se encuentran de pie junto a la puerta y la maestra les llama la atención en lengua wixarika para que reanuden la labor encomendada. La clase finaliza cuando la maestra revisa el producto de sus alumnos.
Con todo ello se puede afirmar que estas estrategias y actividades no contribuyen al logro del conocimiento matemático ya que se prioriza el contenido por sí y no el uso y utilidad que se le puede dar. Para mayor ilustración de la ausencia de la interculturalidad en las prácticas docentes, es evidente la falta de relación entre el conocimiento, como se presenta en los libros de texto y el conocimiento matemático empleado en la comunidad huichol.

En un grupo de tercer grado, en la clase de ciencias naturales, el propósito de la sesión fue conocer las partes que conforman el cuerpo, especialmente cómo está formado y cómo funciona el aparato digestivo ${ }^{3}$. La profesora explica que, como no cuenta con mucho material que aborde los diferentes aparatos, se apoya en una guía de estudio publicada por una casa editorial. Comenta que para introducir el tema de la clase hizo un repaso de otros sistemas y aparatos del cuerpo: "Inicié por ahí, por diferenciar pues ... ya tenían ellos la noción de esos aparatos ..." (ZN3-150206) ${ }^{4}$. En la clase hace uso del pizarrón y de los libros de texto para llevar a cabo la sesión; sobre la clase, sin embargo, es importante mencionar que para comunicarse con los niños utiliza ambas lenguas: el español y el wixarika, de tal manera que algunas preguntas y respuestas que se hacen durante la clase son en español. Es interesante resaltar que los niños se comunican entre ellos en wixarika y cuando se dirigen a la maestra lo hacen principalmente en español.

3 Este es el propósito de la clase que refiere la profesora en la entrevista que se realizó después de la clase.

4 La identificación de entrevistas realizadas después de la observación áulica se hizo mediante un código construido de la siguiente manera: $\mathbb{Z N}$ se refiere a la zona norte, 3 al grado en el que se llevó a cabo la observación áulica y 150206 es la fecha de observación y entrevista después de clase. 
Otra de las estrategias empleadas por la profesora es la escritura que realiza en el pizarrón, mientras que los estudiantes copian lo escrito en sus cuadernos. Esta actividad parece muy común, ya que los niños no hacen preguntas sino que se limitan a la realización de la copia. Los estudiantes se encuentran regularmente callados y sentados en sus lugares, mantienen el libro y el cuaderno abiertos, voltean al pizarrón y escriben; algunos (la minoría) hacen cosas diferentes a la clase y la docente no se percata de ello.

\section{REFLEXIONES FINALES $Y$ SUGERENCIAS}

La visión de la mayoría de los docentes respecto a su práctica está basada en un modelo tradicional de transmisión de conocimientos, en el que el profesor es el protagonista de la clase y el estudiante (alumno) tiene que adoptar un rol pasivo-receptivo para captar y memorizar los conocimientos que se le ofrecen.

Sólo algunos maestros parecen tener una preocupación importante por mejorar su práctica docente con relación a dos puntos críticos: 1) el manejo de grupos multigrados y 2) la metodología para la enseñanza intercultural-bilingüe.

En ese mismo sentido, se puede afirmar que en la práctica docente no se trata de que el profesor cuente con los conocimientos que va a impartir, sino que lo más importante es la capacidad para abordar y lograr que los estudiantes se apropien de los contenidos.

Los docentes se quejan del lento aprendizaje, de los problemas con la lectoescritura y de la falta de motivación de los alumnos; todo esto resulta en un rezago en el cumplimiento de los objetivos del programa de primaria, con bajo desempeño y reprobación. Además, los estándares y las exigencias de los maestros tienden a reducirse y terminan aceptando niveles más bajos de aprovechamiento en sus estudiantes.

Los maestros sienten que están entre dos fuerzas que los empujan y no los apoyan del todo: por un lado, la comunidad, que espera mucho de ellos en su labor docente, y por otro lado, la institución escolar, la SEJ, que en su estructura organizativa de educación indígena (en los mandos medios y altos) no encuentran una articulación apropiada que los apoye con pedagogía y con un programa de educación intercultural bilingüe, adecuado para sus necesidades.

Por todo lo anterior, es necesario integrar un equipo técnico en la Dirección de Educación Indígena de la Secretaría de Educación, en Jalisco, con experiencia y con el conocimiento suficiente para que elaboren un diagnóstico general sobre el estado que guarda la formación indígena, sus formas de trabajo al interior del aula, sus concepciones sobre el ejercicio docente, sus intereses y perspectivas de formación profesional y de trabajo cotidiano.

Indagar cómo concibe el docente los conceptos: docente indígena, educación indígena, bilingüismo, interculturalidad..., ya que son conceptos de uso cotidiano que deben quedar claramente definidos para que se vean reflejados en las acciones que él realiza en la práctica docente.

Realizar seminarios de discusión, más que talleres, y que estos sean impartidos en las cabeceras de las zonas escolares al inicio, a 
mediados y al final del ciclo escolar. Es importante que la temática que se aborde en cada uno de los seminarios tenga una continuidad y un contenido teórico conceptual

\section{REFERENCIAS}

Bernache, G., Esparza, I., Cortés, M. G. y Alcázar, M. (2005, 10 de febrero). [Cuestionario 1. (C1ZN04, C1ZN08, C1ZN11, C1ZN40, C1ZN61, C1ZN76)].

Bernache, G., Esparza, I., Cortés, M. G. y Alcázar, M. (2005). Entrevista individual a profundidad realizada a los profesores que laboran en las escuelas de la zona norte: ZNTXLC35, ZNTXLC37, ZNTXLC38, ZNTBCU27, ZNTBCU40, ZNTBCU43, ZNPNJS07.

Bruner, J. (1990). La educación, puerta de la cultura. Madrid, España: Editorial Aprendizaje Visor.

Bruner, J. (2001). Actos de significado. Más allá de la revolución cognitiva. Madrid, España: Alianza Editorial.

Chavoya, M. L. (1998, julio-diciembre). La educación primaria en tiempos de austeridad. Revista mexicana de investigación educativa, 3 (6).

Delval, J. (2001). La educación, fenómeno humano. En J. Delval (Ed.), Aprender en la vida y en la escuela. Madrid, España: Editorial Morata.

Essomba, M. Á. (1999). Los objetivos de la educación intercultural. Aspectos diferenciales de su función y naturaleza. En M. Á. Essomba (Ed.), Construir la escuela intercultural. Reflexiones y propuestas para progresivo, de manera que sirva como un medio para promover el interés y la asistencia de los docentes. trabajar la diversidad étnica y cultural. Barcelona, España: Graó - Colección Biblioteca de Aula.

Fierro, C., Fortul, B. y Rosas, L. (1999). Transformando la práctica docente. Una propuesta basada en la investigación acción. México: Editorial Paidós.

Geertz, C. (2000). La interpretación de las culturas. Barcelona, España: Editorial Gedisa.

Muñoz, H. (1999). Visión de la educación bilingüe en regiones indígenas de México. México: Universidad Autónoma Metropolitana - Unidad Iztapalapa.

Pérez, Á. (1998). La cultura escolar en la sociedad neoliberal. Madrid, España: Morata.

Postic, M. (2000). Observación y formación de los profesores. España: Editorial Morata.

Sacristán, J. G. (1998). Poderes inestables de la educación. Madrid, España: Morata.

Sacristán, J. G. (2000). La construcción del discurso acerca de la diversidad y sus prácticas. En M. Rovira Gabarro (Ed.). Atención a la diversidad. Caracas, Venezuela y Barcelona, España: Laboratorio educativo y Graó.

Sacristán, J. G. (2001). Educar y convivir en la cultura global. Madrid, España: Morata.

Segovia, D, J. (1998). Educación especial I. Necesidades educativas especiales: una pers- 
pectiva curricular, organizativa y profesional. Madrid, España: Pirámide.

Van Manen, M. (2003). Investigación educativa y experiencia vivida. Ciencia humana para una pedagogía de la acción y la sensibilidad. España: Idea Books.

Vergara, M. (2005). Los significados que tienen los docentes de educación básica respecto a la práctica docente [Tesis doctoral]. México: Universidad La Salle.
Vergara, M. y Bernache, G. (2008). La educación intercultural bilingüe. Estudio de caso en las comunidades indígenas en Jalisco. México: SEP-CONACYT.

Secretaría de Educación Pública. (1993). Plan y programas de estudio 1993. Educación Básica. Primaria. México: SEP.

Registro (ZN2-230506). Realizado por Emilio Carrillo.

Registro (ZN3-150206). Realizado por Ilda Esparza Martínez. 